\title{
An Advanced Heuristic Approach for the Optimization of Patient Flow in Hospital Emergency Department
}

\author{
Oussama Derni \\ Department of Computer Science, University of Mustapha Stambouli, Mascara, 29000, Algeria. \\ E-mail: o.derni@univ-mascara.dz \\ Fatma Boufera \\ Department of Computer Science, University of Mustapha Stambouli, Mascara, 29000, Algeria. \\ E-mail: fboufera@univ-mascara.dz \\ Mohamed Faycal Khelfi \\ RIIR Laboratory- Faculty of Exact and Applied Sciences, Université Oran1 Ahmed Ben-Bella, Oran, 31000, Algeria. \\ E-mail: khelfi.faycal@univ-oran1.dz
}

Received: 27 February 2019; Accepted: 09 April 2019; Published: 08 September 2019

\begin{abstract}
Hospital institutions are one of the most serious organizations over the world, due to their core duty in saving lives, by providing healthcare in an efficient and swift way. Emergency Department (ED) is the main entrance to the hospital, which takes on charge the primary treatment of patients under a time restriction. Many recent studies focused on minimizing the patient Length Of Stay (LOS) by extending resources or altering 'ED' organization (medical teams, scheduling, etc.), without defecting the fundamentals processes. The objective of this study is to improve patient care quality. The improvement is based on resource extending, in order to determine the suitable amount of resource to be added, a Fuzzy Logic system was designed to calculate the target improvement appropriated with the amount of resource and the number of incoming patients. Then, a colored Petri net simulation model was built to measure the reached improvement by comparing it to the current system state. The case study was realized at the 'ED' of Benaouda Benzerdjeb Hospital, located in Oran city, Algeria. As the results of this study, the total patient length of stay inside the 'ED' was minimized, as well as the rate of treated patients.
\end{abstract}

Index Terms-Emergency department, Patient flow, Fuzzy Logic, System modeling, Hierarchical Colored Petri Net, Simulation, Optimization.

\section{INTRODUCTION}

The crucial duty of the emergency departments, which is represented in saving lives by providing a swift and high-quality treatment in a minimal duration, makes it an important field of research through highlighting the various reasons and issues, which affect the 'ED' performances. Emergency department decision makers are facing many challenges, which are growing year after year, with the increasing of the population, the appearance of new diseases with inconsistent symptoms, limited resources, and the difficulty of the prediction of tense situations. Consequently, those facts lead to congestion, a long length of stay, and it extends patient waiting time in the 'ED'. Recently, simulation has become a very flexible alternative modeling approach for the evaluation of competing for health care interventions [1], it helps also to conduct what-if scenarios to test and analyze any proposed solutions before its implementation in the real world. The absence of effective management of patients' flows is one of the central reasons, which leads to 'ED' overcrowding. In the last decades, several researchers such as [2-4] have significantly focused on optimization of patient flows at different stages of the 'ED'.

Actually, the number of individuals who came to the 'ED' in most Algerian hospitals is in increasingly, for a variety of causes, comprising pauperization, growing in pediatric consultations, aging of the population, and an increase in the number of chronic diseases. Furthermore, free and direct admission to this facility and specifically consultation at any time of the day or night, whatever the reason, those things raise the emergency department management challenges. The modeling phase of such a system is required due to the complication of its nature.

In this research, we use a colored Petri net framework for modeling the case study system, in order to lift the lid from the deficiencies of the actual 'ED'. The main objective is presented in the improving of the performance of the considered 'ED', by adding the appropriate amount of resource. However, the determination of the needed amount of resource is not an obvious decision, which in fact depends on the target 
improvement objective. Therefore, we build a Fuzzy Logic system in order to calculate the target improvement based on a set of inputs parameters represented in resources amount and the number of incoming patients.

This paper is organized as follows. The first section presents a literature review. The second section provides a description of the case study 'ED', including patient admission process and the different 'ED' key performance indicators. Section three presents the proposed approach, which includes two main phases. Finally, we provide a general conclusion of the presented work with future perspectives.

\section{RELATED WORKS}

Several recent researches [5-8] in the last twenty years, have lifted the lid from different problems in healthcare systems and focused on providing solutions to those issues, specifically in EDs which they suffer from several phenomena (overcrowding, resources lack, etc.). Different methodologies and techniques in the literature have applied in the process of modeling and optimization of 'ED' systems. Healthcare system investigation has become a necessity to improve its performance over the time, to achieve some goals, such as minimizing the patient length of stay, reducing the charge of healthcare, improving the employment of physical and human resources, and amelioration the care conditions. Simulation is one of the most used methods in operations research, and nowadays it is commonly accepted as an active method to assist management in evaluating different operational substitutions.

An increasing number of studies use simulation in modeling and optimizing EDs systems performances. In the paper of [1], the authors have built a simulation model of the registration procedure in a Chinese hospital based on the collected data, they evaluate the model by analyzing different parameters, then a new model was designed based on the lacks of the old one. In a study [2], researchers have focused on only triage acuity level-3 patients. They apply lean thinking in triage acuity level-3 patients in order to improve emergency department throughput and waiting time. They reached a significant reduction in the process time of discharged from 182 to 160 minutes and transferred patients observation from 186 to 176 minutes. They minimized the total length of stay by 60 minutes, while for the waiting time they achieved a reduction of 23 minutes.

In the work of [9], researchers have accomplished a comprehensive review study to provide an understanding for researchers on 'ED' simulation modeling in terms of presenting gaps to be focused in the future. Authors found that Discrete Event Simulation (DES) modeling methodology is commonly used in the studies, as well many studies focus on patient length of stay, resource utilization and the number of patients discharged as key performance indicators (KPIs) of the 'ED'. Many overcrowding measurement scores have proposed to determine the level of 'ED' overcrowding in an easy and swift way. Among them, we mention Real-time
Emergency Analysis of Demand Indicators (READI) proposed [10] for the prediction of 'ED' demand and resource needs. Besides, there is the National ED Overcrowding Study (NEDOCS) score [11], which is used to evaluate the overcrowding of an 'ED' in an Iranian hospital.

In a study [12], authors evaluate the crowding scores; The Emergency Department Work Index (EDWIN), NEDOCS, and READI by assessing strengths and weaknesses of each score, the evaluations were performed by building a DES model of the 'ED'. Then they used the model results to investigate each of the 'ED' crowding scores under normal working conditions and under two simulated outbreak scenarios in the 'ED'. They conclude that employing both of NEDOCS and EDWIN scores with proposed threshold values could provide a real-time alert for clinicians to anticipate impending crowding. Others [13] have focused on improving time-to-bed to reduce 'ED' congestions; they recognize that unnecessary patient utilization of 'ED' beds and communication interruption are the main reasons causing bed turnaround delays. Authors proposed a creating of a 'Transitional Care Area', where stable conditions patients could be transferred there to spend the rest of their 'ED' visit, as well the use of walkie-talkies to improve communication during the bed turnaround process, which it led to a decreasing of average time-tobed from 120 minutes to 66 minutes.

The use of Fuzzy Logic founds different application in the area of control system design, where human expert knowledge, rather than precise mathematical modeling, of a process or a plant, is used to model/implement the required controller. Uncertainty and ambiguity are evident in many engineering problems. Fuzzy Logic Control (FLC) therefore provides a formal method of translating subjective and imprecise human knowledge into control strategies, thus facilitating better system performance through the exploitation and application of that knowledge. In the paper of [14], the authors study the care provision at hospital emergency departments. They start with a traditional approach, based on queuing theory and simulation models, and then applies a fuzzy approach to the system to analyze the certainty levels provided by the key parameters of the system. In a recent research [15], an integrated fuzzy simulation approach was realized, for the improvement of care quality in an 'ED' of a large general hospital. The simulation model is developed to cover the complete flow of patients in the 'ED'. Authors use a fuzzy simulation for the purpose to find the best nursing schedule based on a minimum patients' queue time.

\section{DESCRIPTION OF THE CASE StUdy ED OF BENAOUdA BENZERDJEB HOSPITAL}

The studied emergency department belong to Benaouda Benzerdjeb Hospital (Oran Hospital University Center) which is one of the biggest hospitals in West of Algeria. The 'ED' receives more than 12000 patients monthly; its doors are open 24 hours a day, 7 days a week. 
It is divided into two main sections; the first one is the Surgical Emergency Service (SES), while the second is the Medical Emergency Service (MES). The SES has a capacity of 26 rooms, distributed in numerous units.

The first floor includes a reception room for administrative procedures; a Vital Emergency Room (VER), which is used for the treatment of patients under shock; two rooms for radiology examinations, actually only one is under use; two examination rooms, one for an ultrasound and the other for a scanner. In the left side of the entry, we found a waiting room and a general surgery room, the last one includes a general surgeon, which has his/her own subspecialty and performs more general work. Critical abdominal pain is the most common emergency presentation requiring surgery since the abdomen has various organs, which may be causing the pain. Moreover, it has a room for orthopedics and traumatology surgery and thoracic and neurosurgical examination rooms. Furthermore, on the same floor, the 'ED' contains a treatment room, fibro scope room, and a visceral unit for patients, who suffering pain from their internal organs, such as bladder, stomach, uterus, or rectum. At the most left side, we found the septic operating room, which includes two operating rooms. This room is the dedicated place to perform a surgical operation, for patients which their states need isolation to prevent the transmission of infection. It exists only one septic operating room in the whole west of Algeria.

The second floor of the studied 'ED' is dedicated to the surgical, and post operations. It includes a treatment room, 14 beds distributed between three rooms for post-surgery' patients before their orientation to another service, and an antiseptic operating room, which includes four operating rooms. The third floor is used for the hospitalization of patients under shock, as well for the hospitalization of medical injury patients. This floor contains 10 hospitalization beds shared between the SES and MES sections.

The second section of the 'ED', which is the MES, it comprises a total of 12 rooms. At the entry, we have a waiting room, which is used simultaneously for the sorting process. Patients waiting for the availability of a general doctor. Inside the MES, we found two medical consultation rooms, one treatment room, and a resuscitator room occupied by a specialist. The MES contains three hospitalization beds for patients under observation for a duration does not extend six hours, plus six hospitalization beds for patients who require a hospitalization period for more than 6 hours.

\section{A. Patient admission flowchart}

Patients' arrival to the 'ED' is either walking (via reception) or by an ambulance. Then, a sorting nurse will sort those patients based on their signs and symptoms. Next, according to their states, she will orient them to the appropriated section of the 'ED'.

In this paper, we considered three main patients' classes; patients in different classes will have a different treatment, thus diverse paths inside the 'ED'. Fig. 1 illustrates the possible paths of patients in the considered
'ED'. The flowchart offers a basis for the discrete event simulation model. It is realized based on the existing patients' flow inside the study system. The different classes of patients are presented as follows:

- Class 1: This class includes patients with a stable state or less urgent state; it represents more than $40 \%$ of the admitted patients based on the collected data of the two last years. Patients of this class are directly consulted by a General Practitioner (GP). Then, depending on each case, the GP may or may not ask for supplementary tests. In the case of the requirement of supplementary tests, patients take the necessary tests and then return to the GP to make its final judgment, before its orientation to another service or discharge to home.

- Class 2: It contains patients who require medical treatments, after the sorting operation, the patient will be oriented to the medical unit. In the case where the patient is in a non-conscious state, she will be admitted in the reanimation unit to stabilize its state. Then, based on a specialist decision, they put her under observation before its discharge or direction to the intensive care unit. In the other case where the patient is in a conscious state, she will be admitted directly into the intensive care unit, before its discharge to home or reorientation to another service.

- Class 3: Patients in a critical case are affected to this class, at the arrival of the patient she will be admitted in the vital emergency room, which includes a set of anesthesiologist and resuscitator doctors. Then, depending on the case of patient, she will be oriented to the medical unit or surgical unit. In the case when a patient needs medical treatment, she will follow the same path of the second class' patients. In the other case when the patient needs surgical treatments, she will be assigned based on her case to the Orthopedics and Traumatology (OTR) unit or to the visceral unit.

\section{B. Emergency department key performance indicators}

In this sub-section, we going to mention briefly the used emergency department key performance indicators (KPIs). The role of the KPIs is to measure the performance of the 'ED', to identify the lacks of the system, which helps in targeting the precise sites to reach a significant improvement of the study system. The following KPIs are the most used in the literature:

- The patient total length of stay: it provides a global idea about the system, it represents the duration of the patient inside the 'ED', from its entrance until its discharge or reorientation. The limit of this indicator is that it does not provide a precise view of system performance at the different stage of patient admission process.

- Leave without being seen (LWBS): it represents the rate of patients, which they enter to the 'ED', 
but left without been seen by a doctor, due to the long waiting time.

- The Door to Doctor Time (DTDT): it is the amount of time between the arrival of the patient and the first medical examination. This indicator clarifies the system performance in the early stage of patient admission.

- Waiting times: taking into account the waiting times of patient at the different stage of its treatments, will provide a precise idea about the care quality and it will help in the selection of the right place to be optimized.

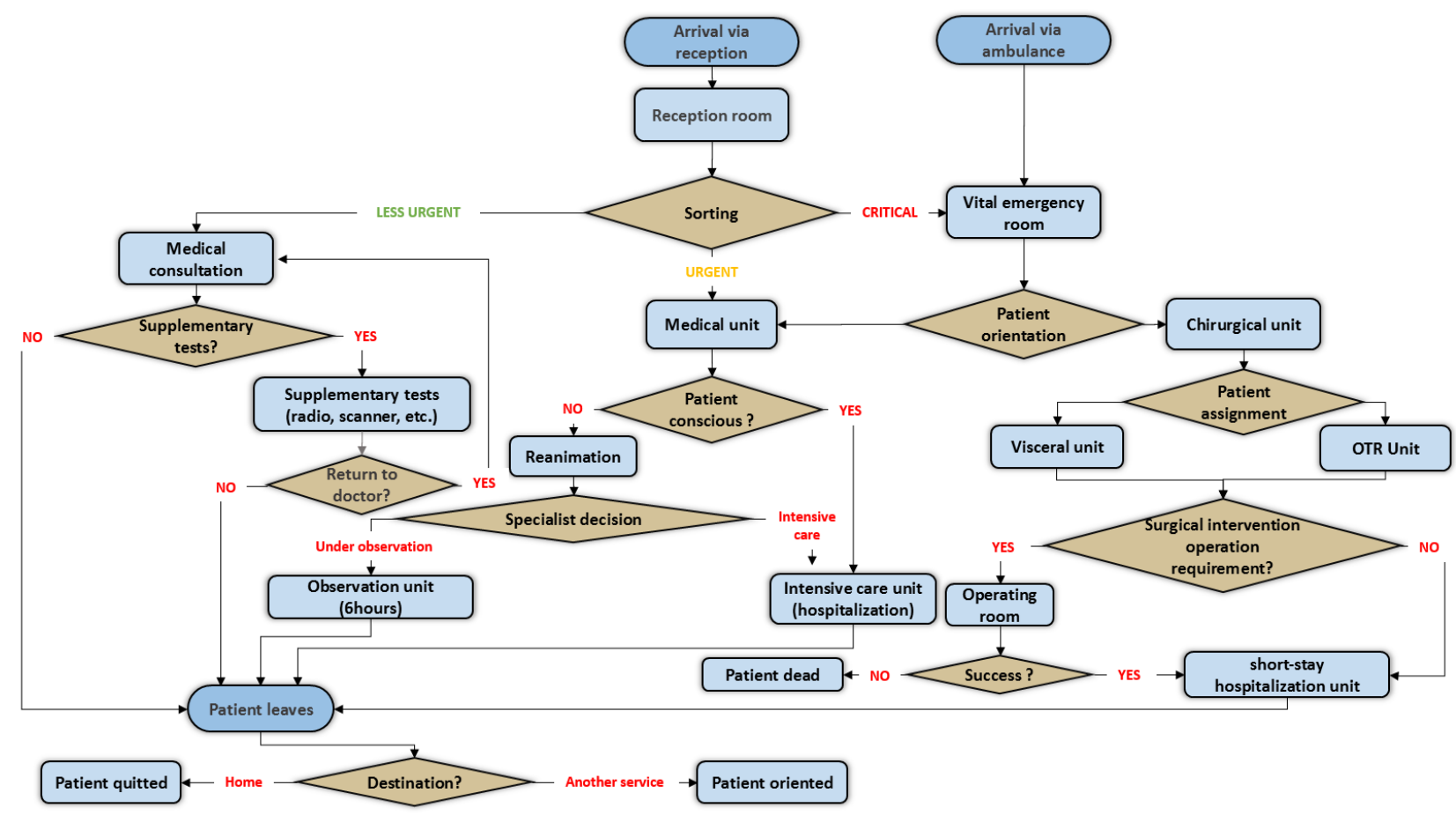

Fig.1. The different paths of patient admission process

\section{THE PROPOSED APPROACH}

In this section, we will discuss our proposed approach for the improvement of patient care quality, which is applied in a case study emergency department. We based on patient total length of stay and the rate of treated patients, as the main key performance indicators to measure the provided care' quality. We will use Fuzzy Logic to demonstrate the relation between the amounts of available resource, the number of incoming patients, the patient total length of stay in the ED and the rate of treated patients, additionally to its help in the calculation of the target output values based on a set of inputs parameters. After the building of the Fuzzy system, we will choose a set of input values appropriated with our objective improvement target. Next, a Colored Petri simulation model will be built to measure the proposed improvement solution, by adding a minimal amount of resources.

A. Phase 1: Measurement of the optimization target values using Fuzzy Logic

In the current work, we use two-input two-output Mamdani-type Fuzzy Logic Controller [16]. We going to design a Fuzzy logic system, which helps us in the determination of the target outputs, based on inputs variables.
In the following, we describe the different stages of the fuzzy control for the appropriated amount of resource to reach a target improvement (Fuzzification, fuzzy inference and Defuzzification).

\section{Fuzzification phase}

During the development of a fuzzy control system, membership functions are defined in three points:

- The general shape of the membership functions (MFs) is based on the particular problem knowledge.

- Some parameters belonging to the membership function remain fixed in order to ensure the correct system behavior in boundary conditions.

- An adjustment method is performed for the remaining parameters.

Each of the above input variables and output variables are converted into its corresponding linguistic fuzzy set.

We have taken two inputs and two output to calculate patients' total length of stay and the rate of treated patients.

\section{a. Inputs fuzzification}

Fig. 2 presents the four membership functions of the Resource amount: low, medium, high and very-high. 
The choice of these membership functions have been fixed after several tests.
While Fig. 3 presents the three membership functions of the Incoming: low, medium and high.

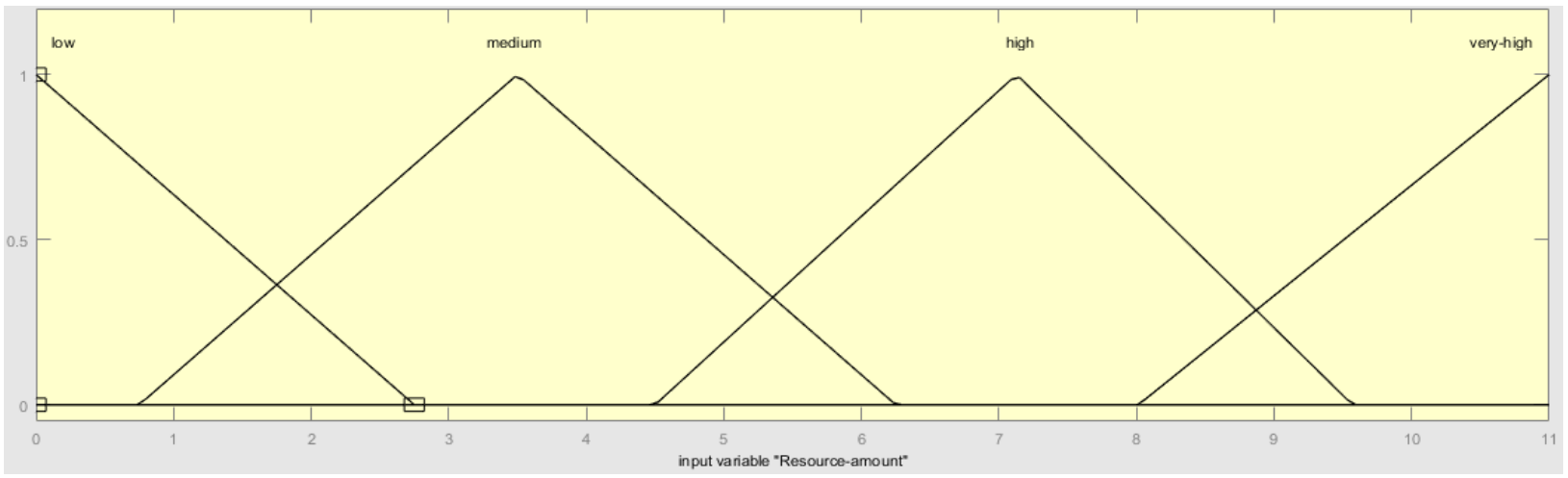

Fig.2. Resource amount' membership function

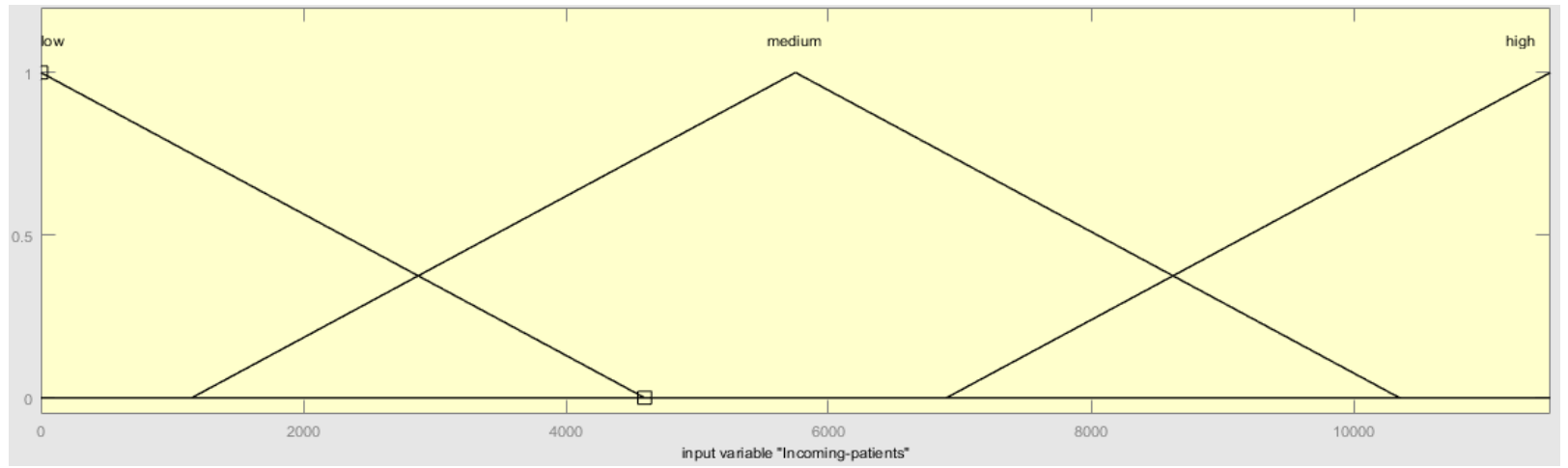

Fig.3. Incoming patients' membership function (estimated for one month)

\section{b. Outputs fuzzification}

The outputs of our fuzzy system are the patient length of stay and the rate of treated patients. Figs. 4 and 5 show their MFs. The patient length of stay is represented by three fuzzy sets: short, medium, and long. While the fuzzy sets for the rate of treated patient are low, medium, and high.

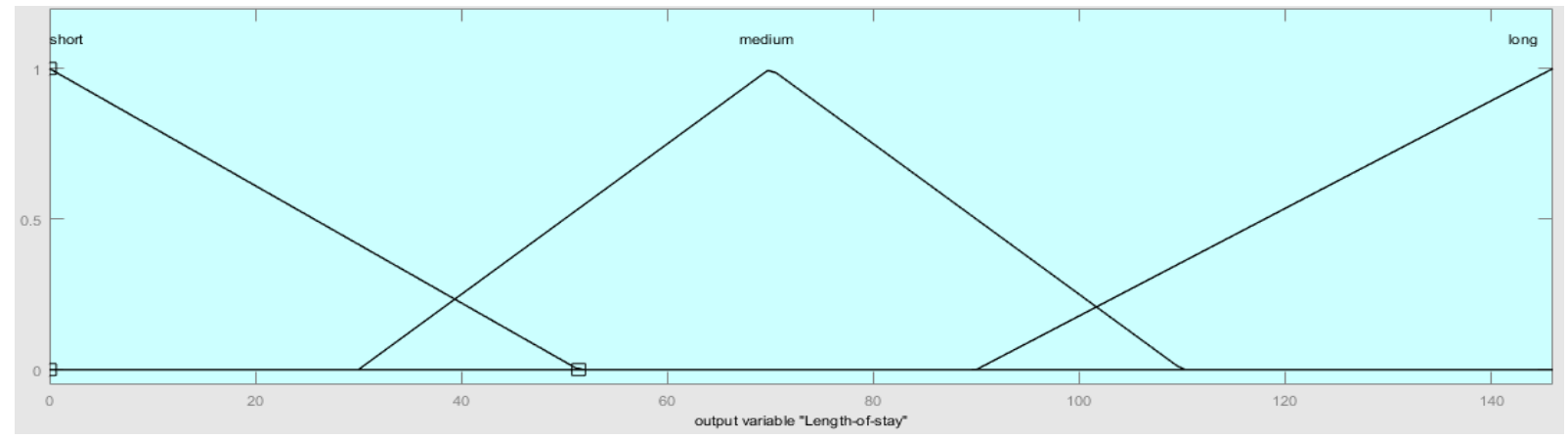

Fig.4. Patient length of stay' membership function 


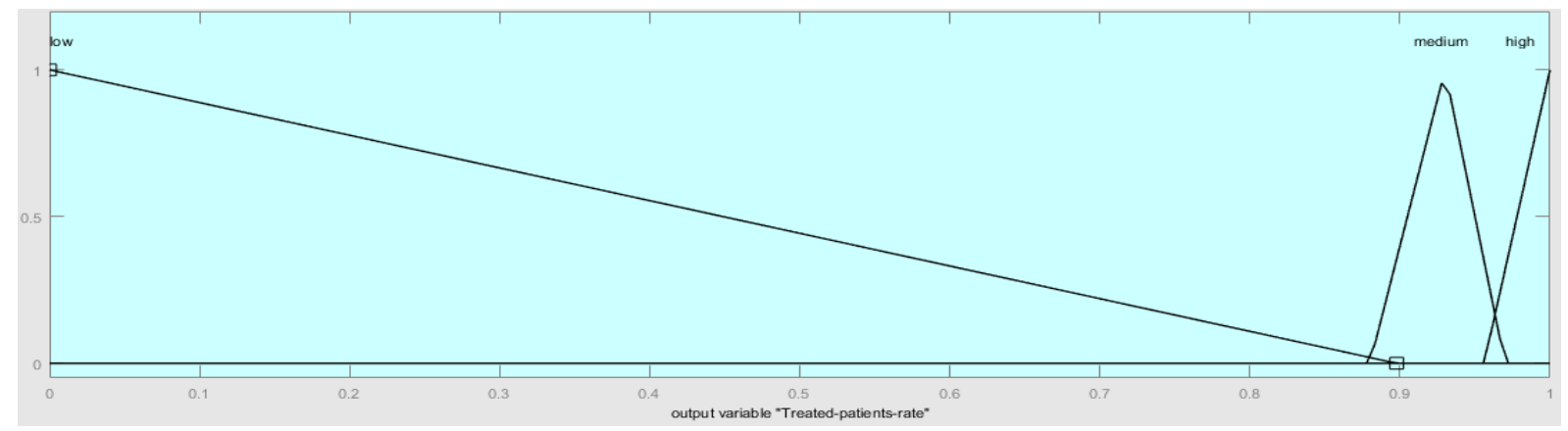

Fig.5. Treated patients rate' membership function

\section{Inference rules}

Mamdani fuzzy inference [17] is the most commonly seen fuzzy methodology and was among the first control systems constructed using fuzzy set theory. In this work, we applied Mamdani rule in the inference engine, which is mainly based on Max-min values as well as maxproducts.
This step presents the inference rules elaboration to determine the patient length of stay and the rate of treated patients. It will be seen later that the number of potential rules of reaction increases directly with the number of labels of the variables. At end we have 12 fuzzy rules. The following table summarizes the rules.

Table 1. Knowledge base

\begin{tabular}{|c|c|c|c|c|c|c|c|}
\hline Rule & \multirow{13}{*}{ if } & $\begin{array}{c}\text { (Resource } \\
\text {-amount) }\end{array}$ & \multirow{13}{*}{ and } & $\begin{array}{l}\text { (Incoming- } \\
\text { patients) }\end{array}$ & \multirow{13}{*}{ then } & $\begin{array}{c}\text { (Length-of- } \\
\text { stay) }\end{array}$ & $\begin{array}{c}\text { (Treated- } \\
\text { patients- } \\
\text { rate) }\end{array}$ \\
\hline 1 & & Low & & Low & & Medium & Medium \\
\hline 2 & & Low & & Medium & & Long & Low \\
\hline 3 & & Low & & High & & Long & Low \\
\hline 4 & & Medium & & Low & & Medium & Medium \\
\hline 5 & & Medium & & Medium & & Medium & Medium \\
\hline 6 & & Medium & & High & & Long & Low \\
\hline 7 & & High & & Low & & Short & High \\
\hline 8 & & High & & Medium & & Medium & Medium \\
\hline 9 & & High & & High & & Medium & Medium \\
\hline 10 & & $\begin{array}{l}\text { Very- } \\
\text { high }\end{array}$ & & Low & & Short & High \\
\hline 11 & & $\begin{array}{l}\text { Very- } \\
\text { high }\end{array}$ & & Medium & & Short & High \\
\hline 12 & & $\begin{array}{l}\text { Very- } \\
\text { high }\end{array}$ & & High & & Medium & Medium \\
\hline
\end{tabular}

\section{Defuzzification phase}

Having put in place the membership functions and inference rules established defining the behavior of the controller, we choose a method of defuzzification. The latter allows transforming the values of fuzzy control domain to the real domain (physical variables). We opted for the defuzzification method called "center of gravity method discreet." This choice is usually conditioned by a compromise between ease of implementation and computational performance.

We have calculated and determinate the target output values using the Fuzzy logic Toolbox of Matlab software.

Figs 6, 7 and 8, illustrate the relationship graphs of the four input and output variables. We can observe that the number of treated patients and the length of stay at the
'ED' depend on the available amount of resource and the number of incoming patients.

Fig. 6 demonstrates the reached improvement represented by the output variables when we increase the resource number and decrease the number of incoming patients. While Fig. 7 shows the current state of the system, which has a capacity of seven resources. In order to reach a significant improvement, ED' managers need to raise the number of available resources to nine instead of seven. Fig. 8 shows the reached output improvements when we increased the number of resource to nine.

In the second phase, we will build a simulation model to measure the impact of a proposed improvement without extending the mentioned amount of resources. 

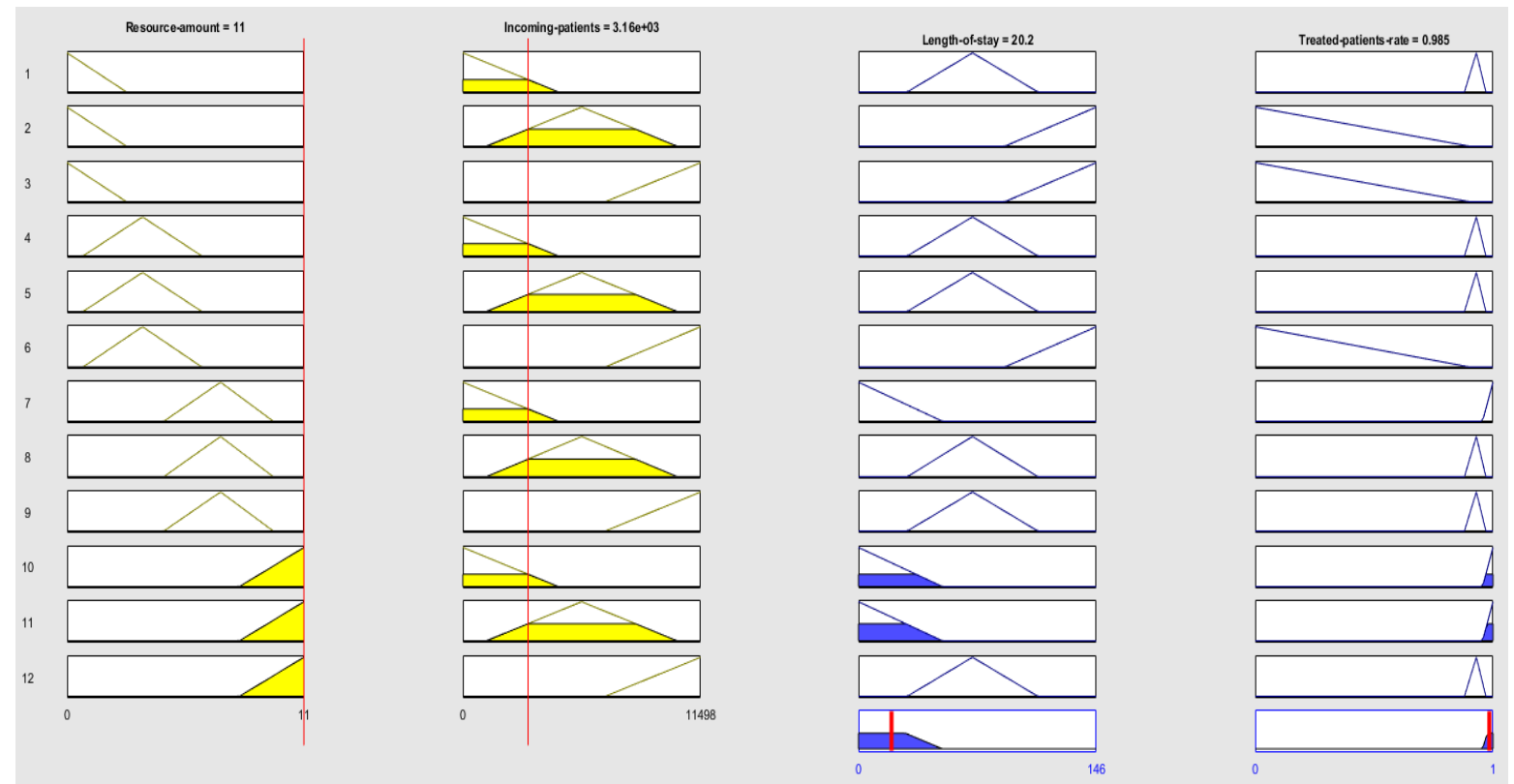

Fig.6. The relationship among Resource amount, Incoming patients, Length of stay and the rate of treated patients
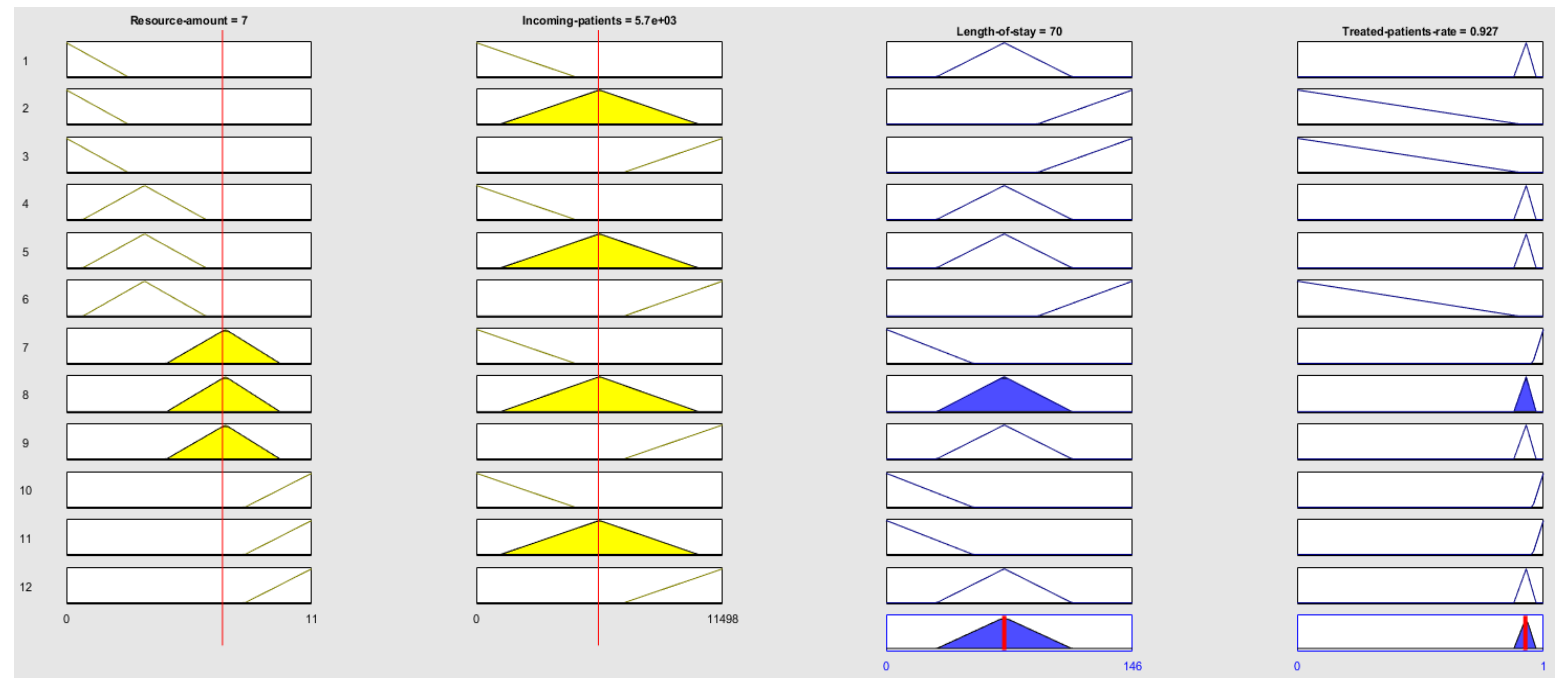

Fig.7. The relationship among Resource amount, Incoming patients, Length of stay and the rate of treated patients of the current system configuration
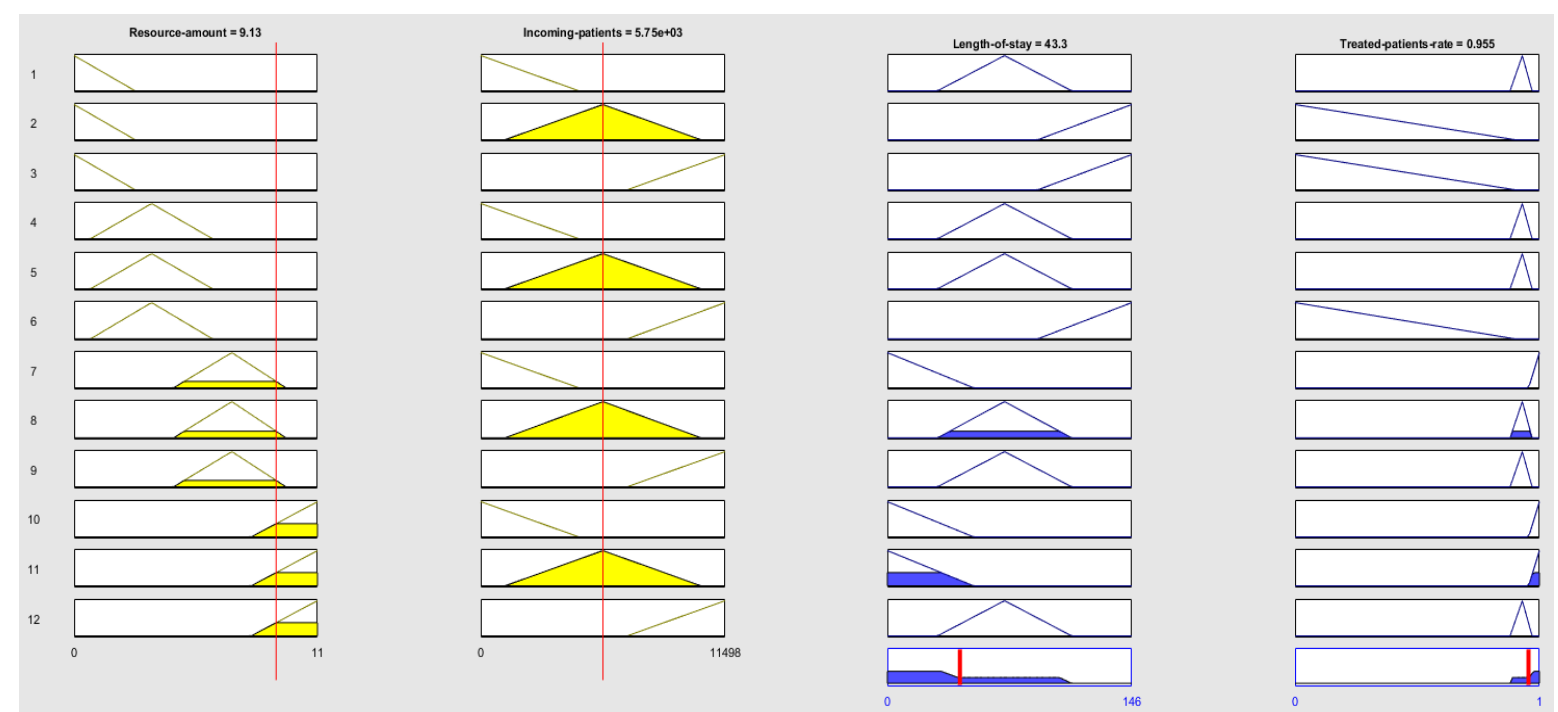

Fig.8. Calculated output values in the case of the augmentation of resources by two units on the current amount 


\section{B. Phase 2: System optimization using Colored Petri nets simulation model}

After the determination of the number of necessary resources needed to reach a significant improvement, we going to propose an improvement solution without exceeding the predefined amount of resources. Then, we measure the system performance after the proposed solution and compare the results with the current system state. For that purpose, we start by modeling the case study system, using a Hierarchical Colored Petri Net (HCPN) [18]. Then, we build a simulation model of the current state of the 'ED' using CPN Tools [19, 20]. The model is designed based on a collected data of one year from the concerned 'ED'. Later, we run many simulations of the current system to compare the results with the collected data, in order to validate the simulation model. Finally, we execute many simulation replications of the current system and the proposed improvement and make a comparison between the results based on a set of KPIs to demonstrate the reached improvement. The following sub-sections will explain in detail the previously described steps.

\section{Simulation model}

In the last decades, simulation becomes a widely spread approach in various research' fields, due to its use to measure the performance of existing and new designed systems before their implementation in the real world. It allows the designer to determine the correctness and efficiency of a design before the system is actually constructed. It offers a way to test different configuration of many systems, without imposing a high cost.

In this study, we build a simulation model of a case study 'ED', the model was constructed based on system observations, and numerous interviews with the medical and administrative staffs of the 'ED'. The model is established and validated to include the core functionalities of the current 'ED' procedures. Hierarchical colored Petri nets were used to model the system, which provides an excellent tool for the modeling of such a dynamic system. CPN Tools is served to build the model, our choice of this tool is due to its extensible functionalities and it provides a powerful set of components for the simulation phase to accomplish the task in a swift and easy way. Fig. 9 shows the main page of our 'ED' model.

The basic stages of patient admission include sorting operation, medical examination, supplementary tests, etc. Each place of the model represents the state of patients' progress inside the system. The patient moves from place to place passing by transitions, which represent the different medical operations and decisions. The exponential distribution function is used to model patient arrival, which is adequate for this case. The patient is modeled by a colored token; it is composed of numerous attributes used for the collection of statistic information about the patient. The model includes a set of places and transitions some of the transitions are double border we called them "substitution transitions". Each of them represents a subnet page of the main page in a hierarchical manner.

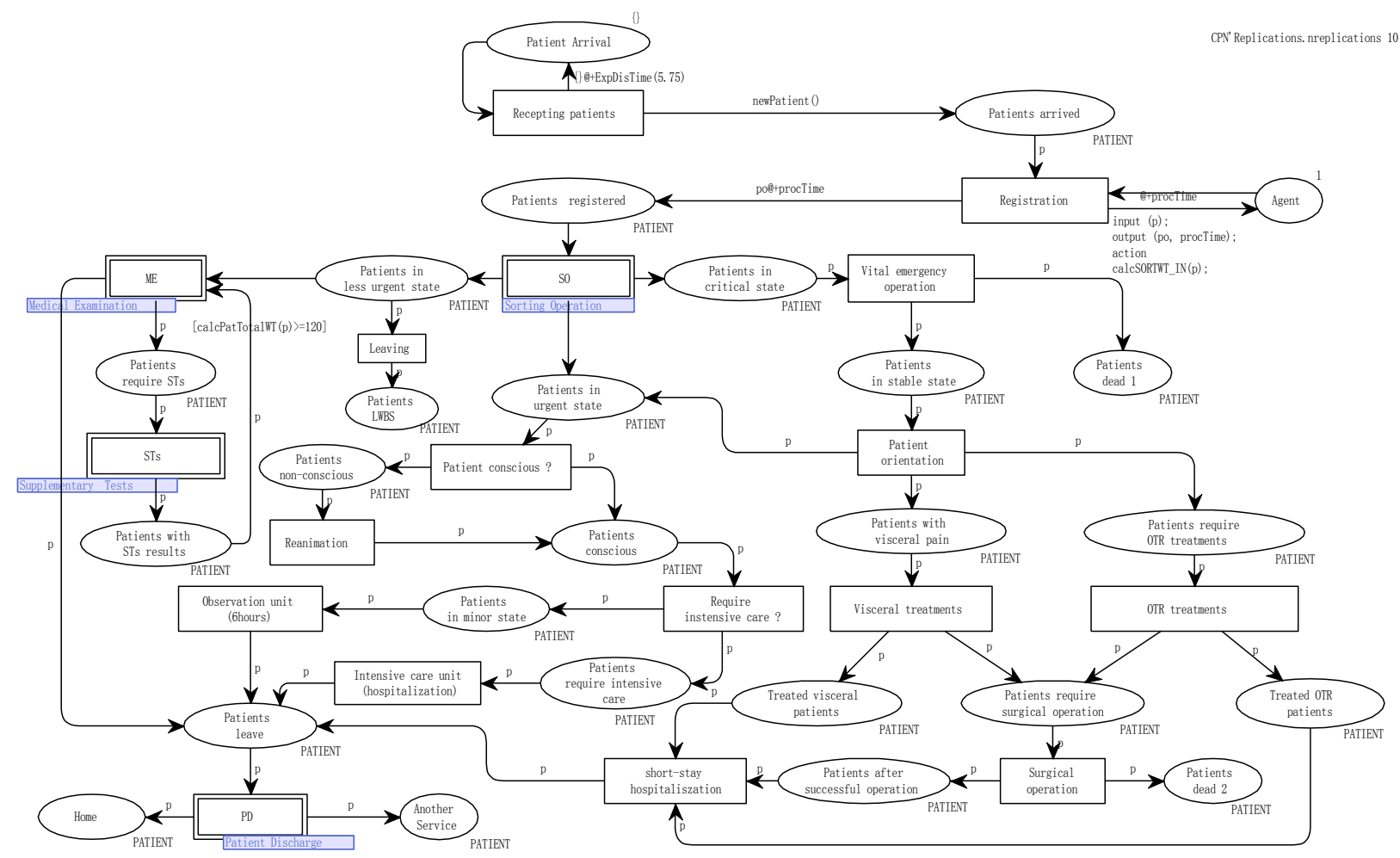

Fig.9. Main page of the simulation model under CPN tools 


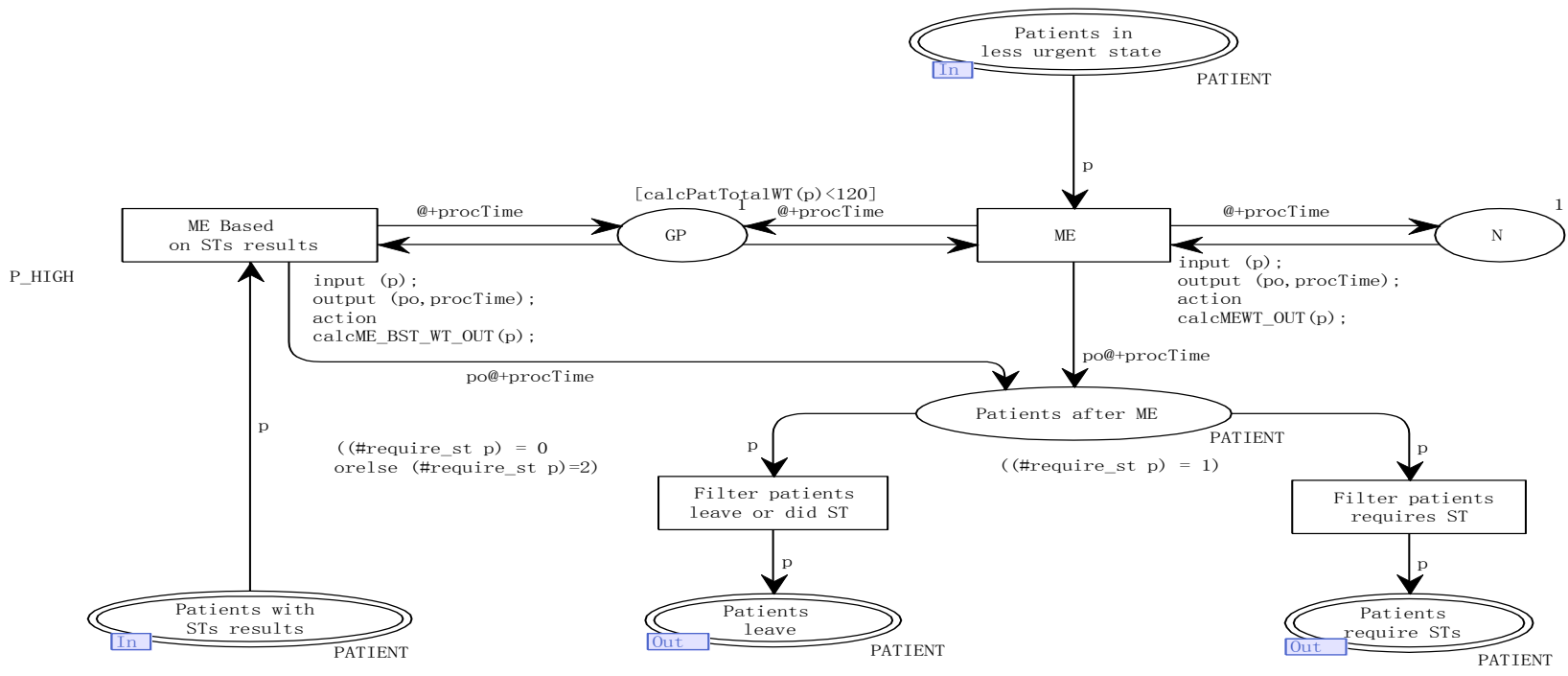

Fig.10. Medical Examination (ME) substitution transition subpage

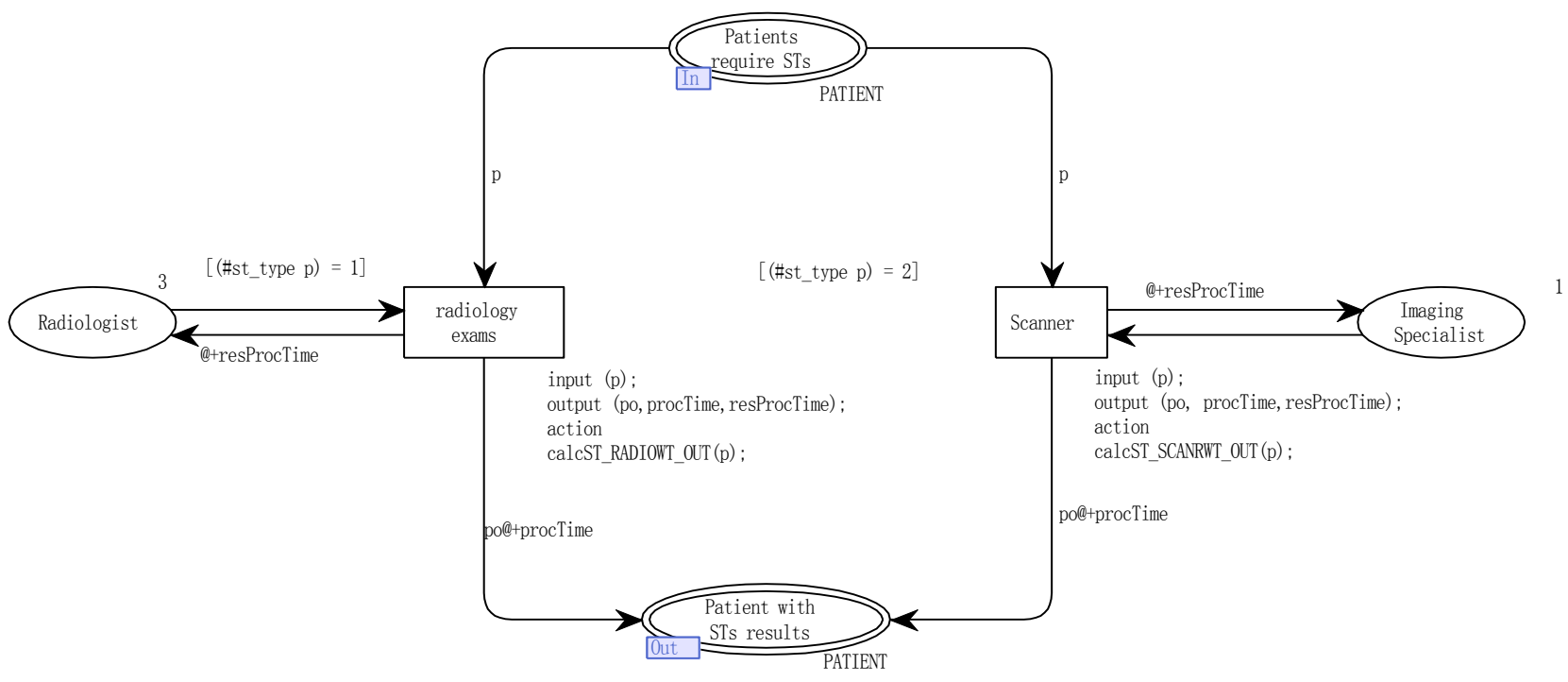

Fig.11. Supplementary tests (STs) substitution transition subpage

As it is exposed in Fig. 9, patients pass by a sorting nurse, which classifies patients based on their states. Then, they will be oriented to the "ME" place for medical examination. Depending on the patient state, she may require supplementary tests, so she moves to the place "STs". The final step is represented in patient discharge to home or transferred to another service.

Figs. 10 and 11 show the substitution transitions of the medical examination and supplementary tests, respectively. Due to the enormous size of the emergency department, we going to focus on the treatment pathway of class 1 patients, which they represent more than $40 \%$ of the admitted patients. Emergency department doctors and staffs validated the proposed model. Additionally, to a comparison between the simulation results of the current system and the collected data (see Table 2).

\section{Proposed improvement and simulation result discussion}

After we built the simulation model using a HCPN, we move on to the phase of system improvement. The main objective is to provide 'ED' managers an efficient solution without a significant increase in the resource amount. The proposed improvement scenario is chosen based on our observations of system' flaws, with the help of domain specialists, without exceeding the amount of resource determined in the first phase. The solution consists of adding two medical staffs in the concerned area of the 'ED', represented in one general practitioner and a nurse. Then, we run the simulation of the proposed scenario for a duration of one month, with ten replications and we take the average results. Table 3 . shows the simulation results of the current system' state with the proposed improvement scenario. For the current system and the proposed scenario, we calculated the key performance indicator, which includes in our case LWBS, DTDT, LOS, waiting times, and tasks durations. The rate of patients that LWBS is minimized by $7.2 \%$, compared to the current system. Additionally, we have reduced door to doctor time from an average of 35.34 to 4.73 minutes, 
which represents an improvement percentage of $87 \%$. Regarding the waiting times, on one side, medical examination waiting time has reduced by $90 \%$ of the actual time. In the other side, due to the decrease of LWBS patients, the waiting time of supplementary tests has slightly increased from an average of 0.57 to 1.21 minutes. The total patient length of stay or LOS provides a global view of system performance. The proposed solution improves the LOS by $43.44 \%$ compared to the existing time.

Table 2. Comparison between the collected data and the simulation results of the current system' state

\begin{tabular}{|l|l|l|}
\hline ED key performance indicators & $\begin{array}{l}\text { Collected } \\
\text { data }\end{array}$ & $\begin{array}{l}\text { Simulation } \\
\text { results }\end{array}$ \\
\hline DTDT (min) & $30-40$ & 35.34751 \\
\hline LWBS (\%) & 7 & 7.2 \\
\hline LOS (min) & $70-75$ & 72.89522 \\
\hline Sorting waiting time & 0 & 0.28704 \\
\hline Medical examination waiting time & $30-40$ & 35.75160 \\
\hline Supplementary tests waiting time & $0-1$ & 0.57505 \\
\hline Sorting duration & $1-2$ & 1.50232 \\
\hline Medical examination duration & $10-20$ & 15.17067 \\
\hline Supplementary tests duration & $40-90$ & 59.44341 \\
\hline
\end{tabular}

Table 3. Simulation results of the current system' state with the proposed improvement scenario

\begin{tabular}{|l|l|l|}
\hline ED key performance indicators & $\begin{array}{l}\text { Benchmark } \\
\text { scenario }\end{array}$ & $\begin{array}{l}\text { Improveme } \\
\text { nt scenario }\end{array}$ \\
\hline DTDT (min) & 35.34751 & 4.73362 \\
\hline LWBS (\%) & 7.2 & 0 \\
\hline LOS (min) & 72.89522 & 41.22892 \\
\hline Total waiting time & 36.23203 & 4.13990 \\
\hline Sorting waiting time & 0.28704 & 0.29797 \\
\hline Medical examination waiting time & 35.75160 & 3.43282 \\
\hline Supplementary tests waiting time & 0.57505 & 1.21098 \\
\hline Total tasks duration & 36.66318 & 37.08901 \\
\hline Sorting duration & 1.50232 & 1.49874 \\
\hline Medical examination duration & 15.17067 & 15.17848 \\
\hline Supplementary tests duration & 59.44341 & 60.42022 \\
\hline
\end{tabular}

\section{CONCLUSION}

In this work, simulation results show the efficiency of the proposed control architecture, which uses Fuzzy controller to implement a hybrid approach based on hierarchical colored Petri nets and a fuzzy controller. The purpose of this combination is to optimize the number of treated patients and to minimize the total patient length of stay inside 'ED'. A Fuzzy logic system was designed to demonstrate the relationships between the amount of resource, number of incoming patients, total patient length of stay and the rate of treated patients. Furthermore, Fuzzy logic provided aid in the determination of the needed amount of resource, based on the calculated outputs values. Then, hierarchical colored Petri net was used to model the system, which helps in the identification of system' issues. Later, a simulation model was built using CPN Tools, in order to measure the impact of a proposed solution. As results, we reached significant improvements of $43.44 \%, 87 \%$ in term of LOS, DTDT, respectively. While the LWBS was minimized by $7.2 \%$. As perspectives, taking into account the different classes of patients (critical, urgent), by applying the same approach will provide an interesting improvement of the system.

\section{REFERENCES}

[1] He, Y., Cai, B., and Wang, M. Research on Optimization of Registration Procedure in Emergency Department Based on System Simulation, Procedia Computer Science, 91:37-46, 2016.

[2] Sánchez, M., Suárez, M., Asenjo, M. and Bragulat, E. Improvement of emergency department patient flow using lean thinking, International Journal for Quality in Health Care, 30(4):250-256, 2018.

[3] Garrett, J. S., Berry, C., Wong, H., Qin, H. and Kline, J. A. The effect of vertical split-flow patient management on emergency department throughput and efficiency, American Journal of Emergency Medicine, 36(9):15811584,2018

[4] Kelley, D. P. and Gravina, N. Every Minute Counts: Using Process Improvement and Performance Feedback to Improve Patient Flow in an Emergency Department, Journal of Organizational Behavior Management, 38(23:234-243, 2018.

[5] Kuo, Y. H., Leung, J. M. Y., Tsoi, K. K. F., Meng, H. M. and Graham, C. A. Embracing Big Data for Simulation Modelling of Emergency Department Processes and Activities, IEEE International Congress on Big Data, New York, 2015, (New York, NY, USA), pp. 313-316.

[6] Marsden, E., Taylor, A., Wallis, M., Craswell, A., Broadbent, M., Barnett, A., Nguyen, K. H., Crilly, J., Johnston, C. and Glenwright, A. A structure, process and outcome evaluation of the Geriatric Emergency Department Intervention model of care: a study protocol, BMC Geriatrics, 17(76):1-8, 2017.

[7] Chang, A.M., Cohen, D. J., Lin, A., Augustine, J., Handel, D. A., Howell, E., Kim, H., Pines, J. M., Schuur, J. D., McConnell, K. J. and Sun, B. C. Hospital Strategies for Reducing Emergency Department Crowding: A MixedMethods Study, Annals of Emergency Medicine, 71(4):497-505, 2017.

[8] Burstein, B., Fauteux-Lamarre, E., Cheng, A., Chalut, D. and Bretholz, A. Simulation and Web-based learning increases utilization of Bier block for forearm fracture reduction in the pediatric emergency department, Canadian Journal of Emergency Medicine, 19(6):434-440, 2016.

[9] Gul, M. and Guneri, A. F. A comprehensive review of emergency department simulation applications for normal and disaster conditions, Computers \& Industrial Engineering, 83:327-344, 2015.

[10] Reeder, T. J., Burleson, D. L. and Garrison, H. G. The Overcrowded Emergency Department: A Comparison of Staff Perceptions, official journal of the Society for Academic Emergency Medicine, 10(10):1059-1064, 2003.

[11] Rahmani, F., Rezazadeh, F., Ala, A., Soleimanpour, M., Esfanjani, R. M. and Soleimanpour, H. Evaluation of Overcrowding of Emergency Department in Imam Reza Hospital in 2015 by Implementing 2 Scales: NEDOCS and EDWIN, Iran Red Crescent Med, 19(6)1-4, 2017.

[12] Ahalt, V., Argon, N. T., Ziya, S., Strickler, J. and Mehrotra, A., Comparison of emergency department crowding scores: a discrete-event simulation approach, Health care management science, 21(1):144-155, 2016. 
[13] Chartier, L. B., Simoes, L., Kuipers, M. and McGovern, B. Improving Emergency Department flow through optimized bed utilization, BMJ quality improvement reports, 5(1):1-6, 2016.

[14] Puente, J., Gómez, A., Parreño, J. and Fuente, D. Applying a fuzzy logic methodology to waiting list management at a hospital emergency unit: a case study, International Journal of Healthcare Technology and Management, 5(6):432-442, 2003.

[15] Azadeh, A., Rouhollah, F., Davoudpour, F. and Mohammadfam, I. Fuzzy modelling and simulation of an emergency department for improvement of nursing schedules with noisy and uncertain inputs, International Journal of Services and Operations Management, 15(1): 58-77, 2013.

[16] Mamdani, E. H. Application of Fuzzy Logic to Approximate Reasoning Using Linguistic Synthesis. IEEE Transactions on Computers, 26(12):1182-1191, 1977.

[17] Mamdani, E. H. and Assilian, S. An Experiment in Linguistic Synthesis with a Fuzzy Logic Controller, International Journal of Man-Machine Studies, 7(1):1-13, 1975.

[18] Jensen, K. Coloured Petri Nets Theory, Basic Concepts, Analysis Methods and Practical Use Volume 1 (Berlin Heidelberg, New York in 1992).

[19] Ratzer, A. V., Wells, L., Lassen, H. M., Laursen, M., Qvortrup, J. F., Stissing, M. S., Westergaard, M., Christensen, S. and Jensen, K. CPN Tools for Editing, Simulating, and Analysing Coloured Petri Nets' in Eindhoven (2003): International Conference on Applications and Theory of Petri Nets and Concurrency, 2003, (Eindhoven, The Netherlands), pp.450-462.

[20] Westergaard, M. and Kristensen, L. M., 'The Access/CPN Framework: A Tool for Interacting with the CPN Tools Simulator' in Paris (2009): International Conference on Applications and Theory of Petri Nets, 2009, (Paris, France), pp.313-322.

\section{Authors' Profiles}

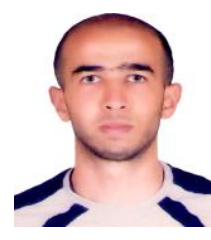

Oussama Derni was born on August 11, 1992. He received the B.Sc. and M.S. degrees from Mustapha Stambouli University, in 2014 and 2016, respectively.

Currently, he is a PhD student of computer science at the University of Mustapha Stambouli, Mascara, Algeria. His current field of research focus on modelling and optimization of socioeconomics organizations. His study interests include, system modeling, flow management, stochastic models, artificial intelligence and discrete-event-simulation. He is also interested in all kind of researches related to optimization and enhancement of dynamic systems.

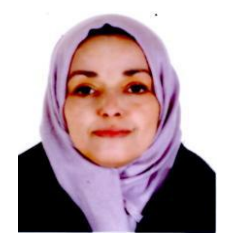

Fatma Boufera received her Engineer degrees in Soft Engineering and in Industrial Computing from Oran University, Computer Science Department, Algeria, in 2000 and 2007, respectively.

She received her $\mathrm{PhD}$ degree from Oran University, Algeria in 2014. She is currently associate professor and research member at Mascara University Institute of Sciences and Technology, Algeria. Her research interests include mobile robotics and artificial intelligence.

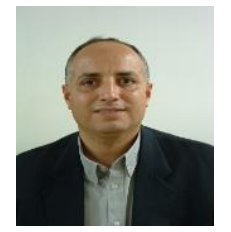

Khelfi Mohamed Fayçal received Ph.D. degree in Automatic Control from Nancy University, France, in 1995. He is currently full rank Professor at the Computer Science Department - Faculty of Exact and Applied Sciences - Université Oran 1 Ahmed Ben Bella - Algeria. He is also a research member at the Laboratory of Research in Industrial Computing and Networks 'RIR Laboratory'. His main research interests include Automatic Control, Industrial Computing, Robotics, and Artificial Intelligence.

How to cite this paper: Oussama Derni, Fatma Boufera, Mohamed Faycal Khelfi, "An Advanced Heuristic Approach for the Optimization of Patient Flow in Hospital Emergency Department", International Journal of Intelligent Systems and Applications(IJISA), Vol.11, No.9, pp.29-39, 2019. DOI: 10.5815/ijisa.2019.09.04 